\title{
Eyeglasses Reduce Risk of COVID-19 Infection
}

\author{
STEVEN LEHRER ${ }^{1 *}$ and PETER RHEINSTEIN ${ }^{2 *}$ \\ ${ }^{1}$ Department of Radiation Oncology, Icahn School of Medicine at Mount Sinai, New York City, NY, U.S.A.; \\ ${ }^{2}$ Severn Health Solutions, Severna Park, MD, U.S.A.
}

\begin{abstract}
Background/Aim: A study of patients in the Hubei Province, China, at the beginning of the pandemic demonstrated that among a group of 276 patients admitted to a hospital with laboratory-confirmed COVID-19, the proportion of patients who said they routinely wore eyeglasses more than 8 hours per day was lower than in the general population. Therefore, wearing eyeglasses more than 8 hours per day may be protective against SARS-CoV-2 infection, possibly because eyeglasses are a barrier that reduces the frequency with which people touch their eyes. The aim of the study was to determine if eyeglasses protect from COVID-19 infection. Materials and Methods: We used UK Biobank (UKB) data to corroborate findings of the Hubei study in the UK population. Results: Eyewear was associated with a reduced risk of infection, odds ratio $(O R)=0.77$. The effects of sex, age, and eyewear were independent and significant at the 95\% level. Men are 1.24times more likely to be infected than women; subjects' risk of infection is less (0.95) for every year of age. Conclusion: The public at large may profit from wearing glasses, as well as wearing face masks and practicing social distancing.
\end{abstract}

Zeng et al. reported on 276 patients in Hubei Province, China admitted to a hospital with laboratory-confirmed COVID-19. The proportion of patients who said they routinely wore eyeglasses more than 8 hours per day was lower than in the general population, suggesting that eyeglasses may offer some protection against COVID-19 (1). We used UK Biobank (UKB) data to corroborate findings of this study in the UK population.

This article is freely accessible online.

*The Authors contributed equally to the conception, writing, and data analysis of this study.

Correspondence to: Dr. Steven Lehrer, Box 1236 Radiation Oncology, Mount Sinai Medical Center, 1 Gustave L. Levy Place, New York 10029, NY, USA. E-mail: steven.lehrer@mssm.edu

Key Words: Eyeglasses, COVID-19 risk.

\section{Materials and Methods}

The UKB consists of more than 500,000 community volunteers aged 40-70 years at baseline (2006-2010), living close to 22 assessment centers in England, Scotland, and Wales. Baseline assessments include demographics, lifestyle, and disease history, with linkages to electronic medical records. Our UK Biobank application was approved as UKB project 57245 (S.L., P.H.R.).

Electronic linkage between UKB records and National Health Service COVID-19 laboratory test results in England are available from March 16 to April 26, 2020, including the peak of daily COVID-19 laboratory-confirmed cases in the current outbreak. During this period, testing of older groups was largely restricted to hospital inpatients with clinical signs of infection, so test positivity is considered a good marker of severe COVID-19 (2).

We analyzed baseline (2006-2010) demographic characteristics and preexisting diagnoses during UKB follow-up. UKB patients with positive COVID 19 tests from England were evaluated. No COVID19 test data were available for UKB assessment centers in Scotland and Wales. We included live subjects who were COVID-19 test positive and deceased COVID-19 subjects who were test positive.

Data processing was performed on Minerva, a Linux mainframe with Centos 7.6, at the Icahn School of Medicine at Mount Sinai. We used the UK Biobank Data Parser (ukbb_parser), a python-based package that allows easy interfacing with the large UK Biobank dataset (3).

$\mathrm{UKB}$ assessed and tabulated eyewear with a touchscreen question (UKB data field 2207): "Do you wear glasses or contact lenses to correct your vision?"; $69 \%$ of people in the UK wear glasses, $13 \%$ wear contact lenses (4). Therefore, $84 \%$ of persons responding 'yes' should have been eyeglass wearers.

\section{Results}

We analyzed data from 12,940 UKB subjects, $52 \%$ female, $48 \%$ male. The mean age was $58 \pm 8.1($ mean \pm SD) .115 (16\%) had died of COVID-19. The subjects were $94 \%$ white British, 3\% Asian or Asian British, 3\% other race.

Eyewear presence or absence in 12,940 patients versus COVID19 infection is shown in Table I. Eyewear was associated with a lower infection incidence (5.2\%) than no eyewear $(10.2 \%, p<0.001$, Fisher exact test two tailed).

Data were analyzed by logistic regression, results in Table II. COVID19 infection, yes or no, was used as dependent variable; sex, age, eyewear, yes or no, were independent variables. Odds ratios (OR) and 95\% confidence intervals were calculated. Eyewear was associated with a reduced risk 
of infection, $\mathrm{OR}=0.77$. The effects of sex, age, and eyewear were independent and significant at the $95 \%$ level. Men are 1.24-times more likely to be infected than women; subjects' risk of infection is less $(0.95)$ for every year of age.

\section{Discussion}

At first glance, the decline in infection rate with age seems counterintuitive. But our analysis is looking only at incidence of infection, which may decrease with age, not at severity or death, which are more common in the elderly. Older people probably stay at home more than younger ones and so are less likely to become infected.

Eye protection plays a vital role during the COVID-19 epidemic. Goggles or a face shield can safeguard eyes and mucous membranes from the virus, which can be spread by virus-containing respiratory droplets, perhaps by airborne viral particles in tiny droplet nuclei, or by touching face or eyes with virus-laden hands (5).

Zeng et al. hypothesized that eyeglasses prevent or discourage wearers from touching their eyes, thus avoiding transfer of the virus from hands to eyes (1). Although eyeglasses do not provide the same eye protection as goggles or a face shield, they may act as a partial viral barrier and diminish viral dose, similar to what has been reported for cloth masks (5).

A weakness in our analysis is our inability to separate eyeglasses from contact lenses. But according to the Centers for Disease Control (CDC), there is no evidence to suggest contact lens wearers are more at risk for acquiring COVID19 than eyeglass wearers (6). Since most UKB responders were eyeglass wearers, and contact lenses do not increase COVID19 susceptibility, our results should be reliable.

The public at large may profit by wearing glasses, as well as wearing face masks and practicing social distancing. Further studies are warranted.

\section{Conflicts of Interest}

The Authors declare no conflicts of interest.

\section{Authors' Contributions}

Dr. Lehrer and Dr. Rheinstein contributed equally to the conception, writing, and data analysis of this study.

\section{Acknowledgements}

This work was supported in part through the computational resources and staff expertise provided by Scientific Computing at the Icahn School of Medicine at Mount Sinai. Research reported in this paper was also supported by the Office of Research Infrastructure of the National Institutes of Health under award numbers S10OD018522 and S10OD026880. The content is solely the responsibility of the Authors and does not necessarily represent the official views of the National Institutes of Health.
Table I. Eyewear in 12,940 patients versus COVID-19 infection.

\begin{tabular}{lccc}
\hline & $\begin{array}{c}\text { COVID-19 } \\
\text { Neg }\end{array}$ & $\begin{array}{c}\text { COVID-19 } \\
\text { Pos }\end{array}$ & $\begin{array}{c}\text { COVID-19 } \\
\text { Infected (\%) }\end{array}$ \\
\hline $\begin{array}{l}\text { No eyewear } \\
\text { Eyewear }\end{array}$ & 1,201 & 136 & $10.2 \%$ \\
\hline
\end{tabular}

Eyewear was associated with a lower infection incidence $(p<0.001$, Fisher exact test two tailed). UKB assessed and tabulated eyewear with a touchscreen question "Do you wear glasses or contact lenses to correct your vision?" $69 \%$ of people in the UK wear glasses, $13 \%$ wear contact lenses, according to the College of Optometrists. According to the Centers for Disease Control (CDC), there is no evidence to suggest contact lens wearers are more at risk for acquiring COVID-19 than eyeglass wearers.

Table II. Data analyzed by logistic regression.

\begin{tabular}{lccccr}
\hline & OR & $95 \% \mathrm{LB}$ & $95 \% \mathrm{UB}$ & $p$-Value \\
\hline Gender & 1.24 & 1.06 & - & 1.44 & 0.005 \\
Age & 0.95 & 0.94 & - & 0.96 & $<0.001$ \\
Eyewear & 0.77 & 0.62 & - & 0.96 & 0.018 \\
\hline
\end{tabular}

COVID-19 infection, yes or no, was used as dependent variable; sex, age, eyewear (yes or no) were independent variables. Odds ratios (OR) and 95\% confidence intervals were calculated. LB, lower bound; UB, upper bound. Eyewear is associated with a reduced risk of infection, 0.77 . The effects of sex, age, and eyewear were independent and significant at the $95 \%$ level. OR indicates that men are 1.24 times more likely to be infected than women; subjects' risk of infection is less (0.95) for every year of age.

\section{References}

1 Zeng W, Wang X, Li J, Yang Y, Qiu X, Song P, Xu J and Wei Y: Association of daily wear of eyeglasses with susceptibility to Coronavirus disease 2019 infection. JAMA Ophthalmol: 138(11): 11961199, 2020. PMID: 32936214. DOI: 10.1001/jamaophthalmol.2020.3906

2 Atkins JL, Masoli JAH, Delgado J, Pilling LC, Kuo CL, Kuchel GA and Melzer D: Preexisting comorbidities predicting COVID-19 and mortality in the UK biobank community cohort. J Gerontol A Biol Sci Med Sci 75(11): 2224-2230, 2020. PMID: 32687551. DOI: $10.1093 /$ gerona/glaa183

3 Zhu A, Salminen LE,Thompson PM and Jahanshad N: The UK biobank data parser: A tool with built in and customizable filters for brain studies. Organization for Human Brain Mapping Rome, Italy, June 9-13, 2019. Available at: https://github.com/USCIGC/ukbb_parser [Last accessed on March 5, 2021]

4 Britain's eye health in focus - Wales council of the blind. The college of Optometrists, 1-19, 2013. Available at: http://www.wcbccd.org.uk/perspectif/library/BEH_Report_FINAL\%20(1).pdf [Last accessed on March 5, 2021]

5 Maragakis LL: Eye protection and the risk of Coronavirus disease 2019: Does wearing eye protection mitigate risk in public, nonhealth care settings? JAMA Ophthalmol: 2020. PMID: 32936218. DOI: $10.1001 /$ jamaophthalmol.2020.3909

6 CDC: Protect your eyes. Available at: https://www.cdc.gov/ contactlenses/protect-your-eyes.html [Last accessed on March 5, 2021]

Received February 9, 2021

Revised February 23, 2021 Accepted March 5, 2021 